\title{
Study of the behaviour of the shutter prototype for non-residential buildings, aimed at containing the summer heating load
}

\author{
S. Grignaffini, S. Cappellanti \& A. Cefalo \\ Department of Technical Physics, University of Rome, "Sapienza”, Italy
}

\begin{abstract}
This research aims at realising a shutter prototype which would allow the containment of the summer heating load in non-residential buildings. With its positioning at the height of the envisaged holes and its particular geometrical shape, this prototype will replace the standard vertical shutter with one containing a glassblock with a sharp inclination with respect to the incidence of sun rays in the summer time.

A measurement campaign is envisaged in order to optimize the glass inclination with respect to the incidence of sun rays both during the winter and summer time and the width of the horizontal anti-dazzle screen placed at the top of the shutter.

The lack of mobile or steady anti-dazzle screens ensures a perfect view of the external surroundings; in addition to this, the standard levels envisaged for by the existing regulations on natural lighting on the back wall will not be altered.

The aim of this work is that of providing a passive element which could concretely guarantee a high level of acoustic and visual comfort, and a proper ventilation in indoor non-residential environments.

Keywords: shutter, heating load, acoustic and visual comfort.
\end{abstract}

\section{Introduction}

This study is about the widening of a particular augmentation system of energetic efficiency in thermal area, by the creation of a building component that can be reproduced in series and integrated in the realization of buildings for nonresidential use. 
By using appropriate bioclimatic technologies, it is possible to decrease the interior summery thermal load, responsible for the overheating. The outcomes of the activity developed are the natural reduction of the internal volume temperatures and the consequent reduction of the energy required to guarantee thermohygrometric comfort conditions, suitable to the current standards. Big glassed surfaces to South are the solution proposed in the bioclimatic field to solve the problem of winter heating. The problem of summery overheating is overlooked, due to the absence of solar radiation defence systems.

This article tackles the four concepts in bioclimatic planning and their integration in window design: an adequate plan of the glassed surfaces, the cooling technological systems, the screenings and shutters and frames.

The prototype consists in an exterior aluminium frame, containing a fixture (or section) and a double-glazing in overhang to the front. The upturned triangular shape, with the minor base placed in the upper part of the area, will give the glassed surface an inclination on the incidence of sunbeams, with respect to a vertical glassed surface. The overhanged upper part contains a technological system of mechanical channels and fans, placed in order to provide the automatic introduction and extraction of the air to guarantee an appropriate change of air and to ensure the required thermal comfort. In the upper part, called "cassettone" (coffer), there are external movable screenings, placed and designed to provide the right shading to reduce the interior temperature, during the main summery hours.

\subsection{Transient calculation}

The research activity with applicative aims, follows a study in the mathematical technical physical field on heat transmission, a phenomenon explained by the equation of Fourier that provides for the dependence from the physical parameter time, needing an analysis in the transient state.

$$
D \nabla^{2} T=\frac{\partial T}{\partial t}
$$

This kind of complex analysis provides more reliable outcomes, especially in the bioclimatic field, where the quantities are influenced by the variable time.

\section{The glass inclination}

The glassed surface will be placed with an inclination angle to the vertical unlike the zero, in order to exploit the different attitudes of the glass on the solar radiations, regarding the angle of incidence.

A glassed surface, basically, is a body able to absorb and reflect solar radiation, and also able to let it pass in certain quantities. This physical statement is summarizable in the identification of three coefficients $(a, r, t)$, variable according to the solar radiation angle of incidence (see figure 2 ).

By developing this intrinsic capacity of the material, we have optimized a component present in every building: the window, showing (for Rome, without 
Eco-Architecture II 265

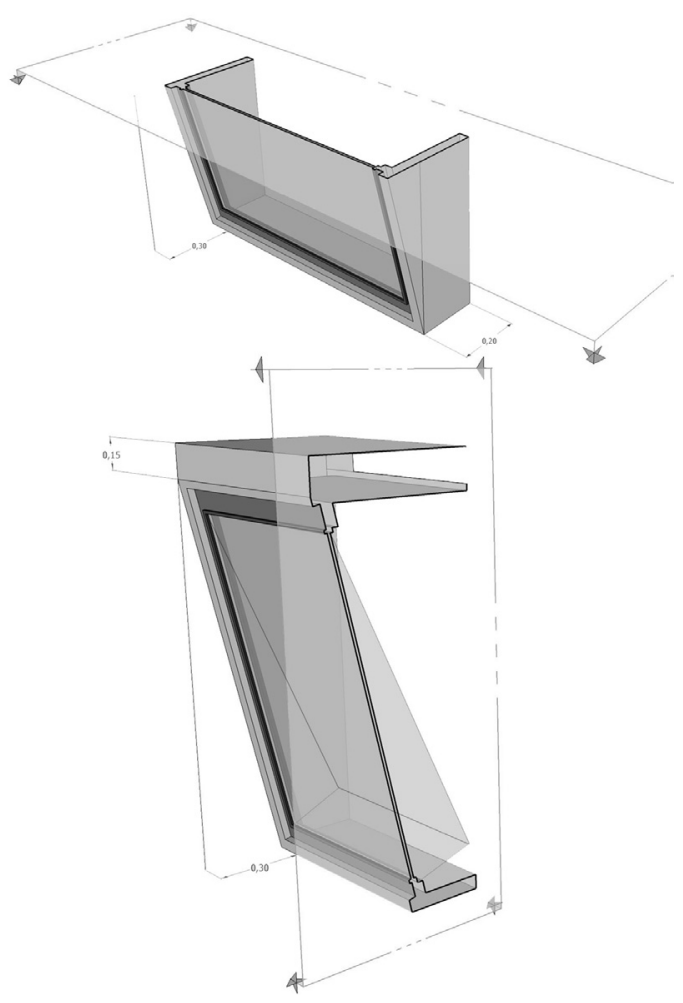

Figure 1: Rendering 3D of window prototype.

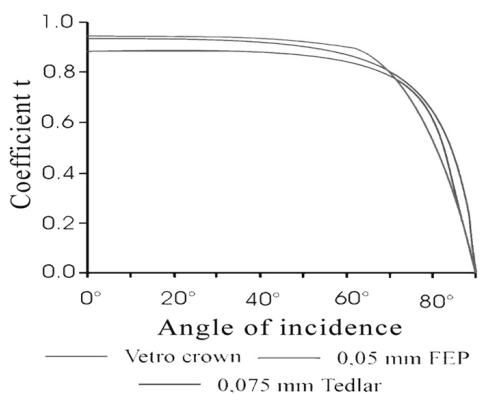

Figure 2: Transparency coefficient at the variation of angle of incidence.

losing a general validity) that, by regulating the inclination of the glassed surface, it is possible to get, to a little disadvantage during the winter, more advantages during the summer. 
Then, the problem is how to reach the lower amount of energy required for the maintenance of thermo-hygrometrical comfort conditions, during the entire calendar years (warming period + no intervention period + cooling period).

\subsection{Planning aspects}

\subsubsection{Geometry}

We have solved the problem of geometrical optimization to reach the highest energetic efficiency, by changing the parameter of inclination, in regard to the vertical, on a window model, during simulations in transient statement. The outcomes indicate the disposition of the glassed surface with an inclination of $11^{\circ}$ to the exterior. We specify that these glassed surface fixtures were $\mathrm{m}^{2} 2.26$, orthogonal to position forms with the South.

To reduce the external exchange surface, we put the lower part of the window flush and overhung of $30 \mathrm{~cm}$ the upper one, integrating it with two specific technological systems, suited to avoid overheating.

\subsubsection{Glassed surface}

The thermal cutting profilats (or fixtures) are made of two parts, internal and external, split and linked by little bars of reinforced polyamide with fibre glass. This very resistant material keeps unaltered the stability of the profilat and acts as insulation between the two metallic parts. The thermal cutting contains the loss of heat by direct transmission and reduces sweat formation. The double glazing is constituted by two or more sheets linked to the perimeter by anodised aluminium, containing a special dehydrator to get among the sheets a dehydrated air space. A plastic perimetrical sealing prevents air exchanges from outside.

\subsection{Simulations}

By a software for the calculation in transient state, we have studied how to solve numerically the problem of optimization of the glassed surface inclination angle. The main output quantity is the $\dot{Q}_{s}$ "Rate of solar energy gain through window", achievable by the following function:

$$
\dot{Q}_{s}=A \tau_{b} I_{b T}
$$

where $A$ is the area of the glassed surface, $\tau_{b}$ the transmittance of incident beam radiation and $I_{b T}$ the beam solar radiation incident on window. We have also to explain that transmittance of beam and diffuse radiation are considered separately $\tau_{b}$ and $\tau_{60 *}$ (transmittance of incident diffuse and ground reflected radiation) are determined using function subroutine TALF in software.

The experimental numeric analysis on the glass inclination showed a latitude and climatic dependence, making impossible the research of a total optimization. Such research is complicated by the interaction of the window with the building. In the main outcomes we will be, above all, underlined the conceptual confirmation we got. 


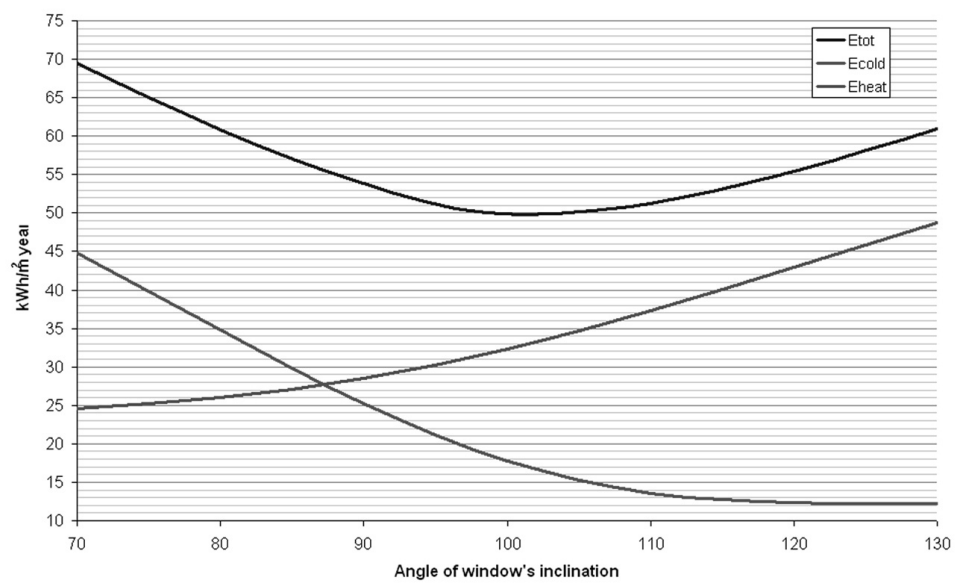

Figure 3: Energetic efficiency according to the inclination.

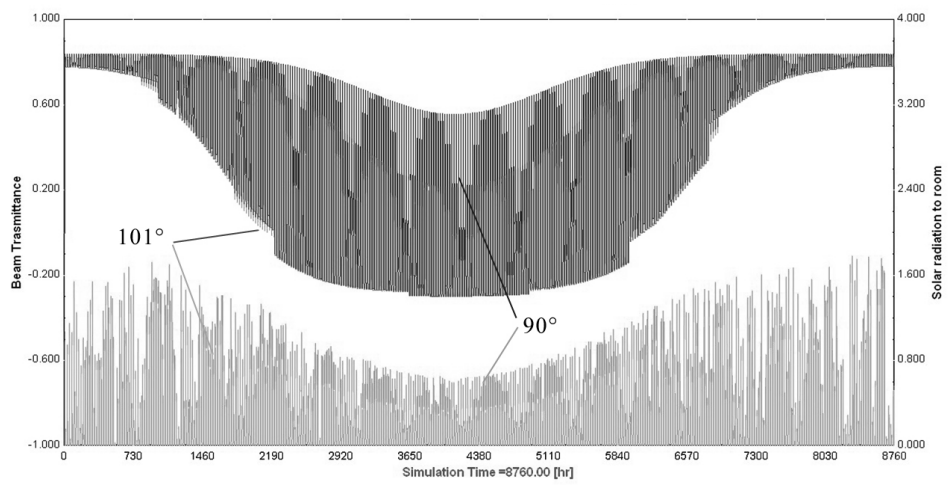

Figure 4: Output transparency coefficient to different inclinations.

In figure 3, you can see how 4 windows in a room of $70 \mathrm{~m}^{2}$ south-facing influence the energetic efficiency in different ways, according to their inclination. The larger is the glass inclination angle, the larger will be the heating energy required and the less will be cooling one. The total energy has a minimum, which in this case is $10^{\circ}$ to the outside. On the other hand, the study using as planning parameter the coefficient of transparency and the solar radiant energy entering is significant, as shown in figure 4 , where the outputs have an inclination of $90^{\circ} \mathrm{e}$ $101^{\circ}$. When a glassed surface is properly inclined, the consumptions decrease by $15 \%$. 


\section{Night-cooling}

The high temperature ranges between day and night require a system that allows the cooling of the rooms, without a big waste of energy. The solution could be the use of external air as natural convector fluid through mechanical ventilation systems that move masses of air from the outside in the inside, providing an appropriate mixing of the two masses at different temperatures.

\subsection{Component integration}

Inside the above mentioned coffer, there is a mechanical ventilation system realized through the installation of 2 circular channels, with a diameter of $10 \mathrm{~cm}$ for a section of 78,5 $\mathrm{m}^{2}$, each one for an area of $0,01576 \mathrm{~m}^{2}$. Considering the mean air speed of $5 \mathrm{~m} / \mathrm{s}$, the capacity of the entering air for each window is $282,6 \mathrm{~m}^{3} / \mathrm{h}$.

These values come from the application of a thermal study, aimed to guarantee a change of air in every room during the night, on a step-surface of $25 \mathrm{~m}^{2}$ ( $h=3 \mathrm{~m}$ ), equivalent to 3 times the volume/hour. This value is enough to guarantee a significant decrease of the internal temperatures during the night. This aspect, along with a thermal inertia in the building, allows a significant temperature decrease $\left(3\right.$ or $4^{\circ} \mathrm{C}$ ) during the day, too. In figure 5 , the system dimensioning is shown, by changing the number and the diameter of the channel and the mean air speed.

Concerning the facility dimensioning, you can refer to the following report that provides the diameter $D[\mathrm{~cm}]$ of one pipe, with the influence volume $V\left[\mathrm{~m}^{3}\right]$, the

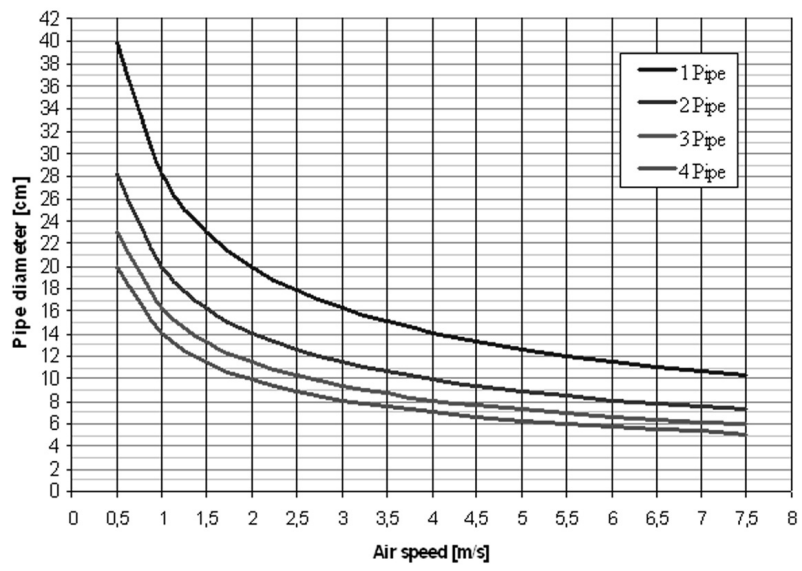

Figure 5: Ventilation facility dimensioning for the night cooling. 


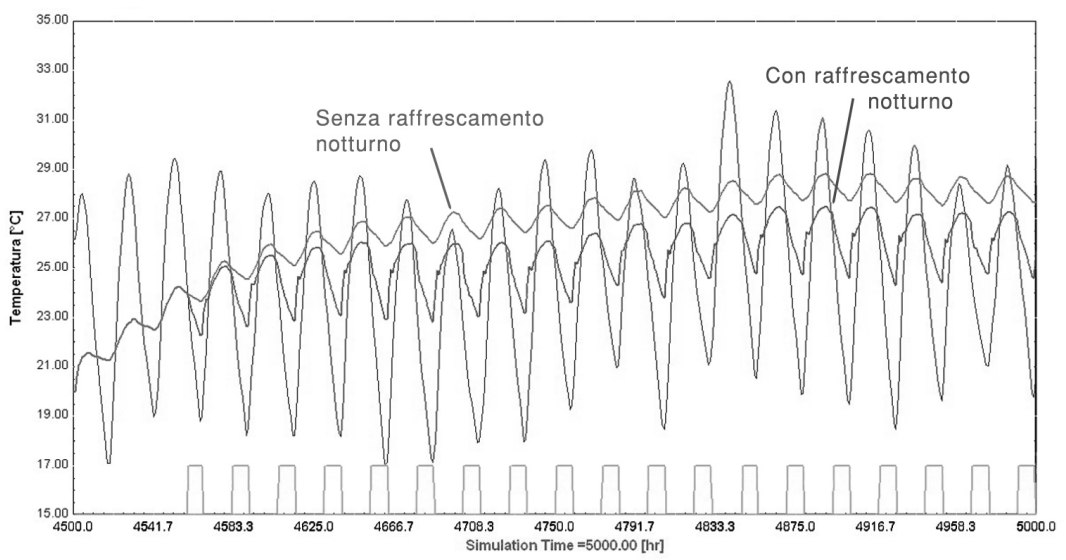

Figure 6: Outcomes of the summer nocturnal ventilation $(3 / \mathrm{h})$ automatic system.

number of changes each hour $n_{r}$ and of the channels $n_{p}$, the speed $v[\mathrm{~m} / \mathrm{s}]$ :

$$
D=1,881 \sqrt{\frac{V n_{r}}{v n_{p}}}
$$

The outcomes are in figure 5 for the project of this research.

\subsection{Simulations}

Simulations on possibilities and effects of the nocturnal ventilation, during the summer.

Figure 6, shows the simulation on a bioclimatic building, provided with a nocturnal cooling system. The system automatically verifies three parameters: the external temperature, the internal one and the weather. The cooling starts constantly (3/h volume) in certain night hours (0-6 and 21-24), each time the external temperature is lower than the internal one and the internal temperature is higher than $23^{\circ} \mathrm{C}$ and it stops when the internal temperature reaches the $19^{\circ} \mathrm{C}$.

The difference between the temperature with or without cooling by nocturnal ventilation is underlined. The internal temperature, in case of ventilation in a short time, gets near to the external one, cooling by transferring the heat accumulated during the day.

\section{Movable screenings}

To avoid overheating owing to the excessive thermal load of solar radiation on the glassed surface, a screening system is needed, able to let the solar irradiation in during the winter, and reflect it during the summer. Besides, it should guarantee a natural illumination all the year. 


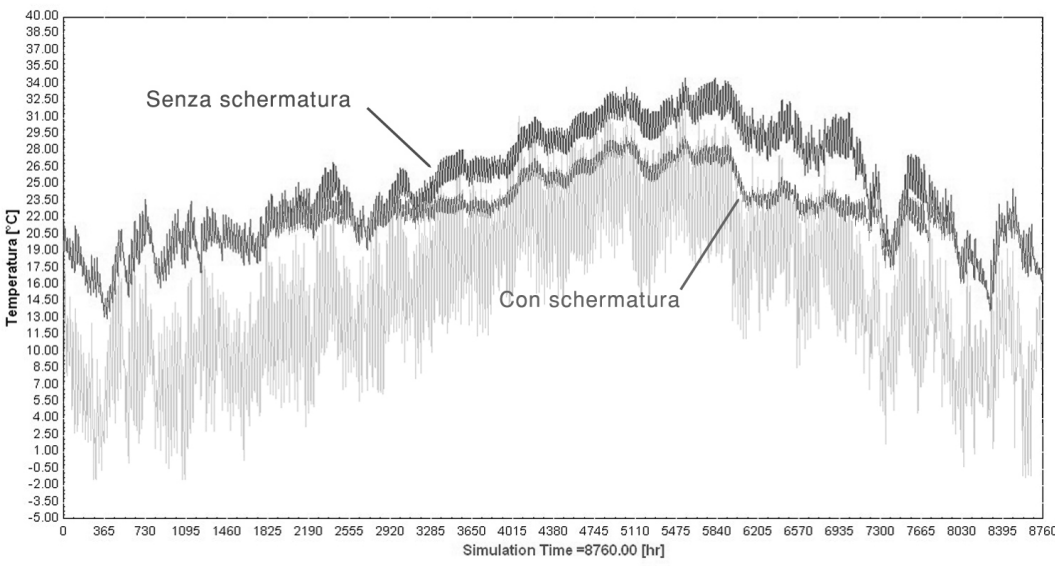

Figure 7: Influence on the movable screening temperature.

To develop these functions, the following conditions are necessary:

- external disposition. In fact, if placed inside the screening effect would be null;

- adaptability of the element to the solar incidence angle.

Then, we can say that the external screenings, for their versatility, are preferred to the static ones. The above mentioned system would improve if provided with an automatic and independent system that manage the opening and the closing of the slats.

\subsection{Integration in the component}

The simulations done suggest the integration of a movable screening system, which would be placed externally the foldaway glassed surface, in the front of the coffer.

The system proposed is the Venetian one, for its versatility in getting a dark surface by the position and disposition of the slats.

\subsection{Simulations}

An appropriate movable screening system can improve the internal environment, by guaranteeing a decrease of the internal temperature, in the absence of a thermal control system. Figure 7 shows how, during the needed periods, a temporary glassed surface screening of $60 \%$ can modify the internal temperature in an environment of $70 \mathrm{~m}^{2}$, with 4 windows south-facing. 


\section{High efficiency frame}

There wouldn't be any outcomes if the quality of the framing system thermal insulation of the glassed surface had not been considered. In fact, the frame is the most susceptible to the heat bridge, exposing the entire system to significant losses of energy, when high transmittance frames are used.

Then, we have studied a high efficiency framing system to the heat bridge.

The planning has been complicated by the authors, who wanted to realize a system that allowed the placement on the punching, in order to save workmen and time.

\subsection{Planning features}

The structure is realized in aluminium (lightness, corrosion resistance, lastingness, low maintenance, easy extrusion of sections), steel (structural endurance), wood (ecological insulating and esthetic quality), insulating foams (high thermal resistance).

The frame junctures, containing the double glazing, leave a small gap on the outside of the two sections. The air passes through this gap, and pushes off a fin shaped seal, placed on the fixed frame against the wall where the mobile casement is placed so that the stronger the wind is the more the seal adheres to the frame improving the result. The absolute inalterability and the mechanical coupling of the aluminum frames keep the sealing unchanged in time.

\subsubsection{Opening}

The envisaged frame also allows the inward opening allowing an occasional air exchange which is necessary for the health of environments in particular situations.

Under a more practical point of view, the opening is realized through the already used system Wasitess, but in a reverse manner, that is with an opening from the lower area inward with a horizontal axis based rotation seal on top of the glass surface. The rotation angle, limited to the length of the axis, lies between $0^{\circ}-10^{\circ}$ in order to guarantee a minimal, and not excessive, intake of air not controlled from the outside.

\subsubsection{Placement}

Finally, the placement of the prototype will happen through the anchorage to the structure from the outside. The contact area with the building will use the advantage of the integration between the frame and the thermo-insulating material of the building's seals.

\section{Conclusions}

The window prototype which results from a more complex research area within the standards of the bioclimatic planning shows, even in its preliminary stage, how 
it has been possible to obtain a real reduction of the energetic costs quantifiable in the $\mathrm{Kw} / \mathrm{h} \mathrm{m}^{2}$ needed for the maintenance of thermo-moist comfort conditions.

The obtained results come out of the integration of only three bioclimatic planning concepts analysed and adapted to a specific climatic situation.

The authors of this article will complete, at a future stage, the work started here with further developments (result of their continuous research in the area) such as, the theoretical numerical aspect, the practical feature and the economic evaluation aspect.

\section{References}

[1] Antonio Cefalo, Progettazione bioclimatica: stato dell'arte e verfiche in regime stazionario e transitorio, Degree thesis in Civil Engineering - University of Rome "Sapienza", 2007.

[2] Simone Cappellanti, Progettazione bioclimatica il comfort termico mediate l'uso razionale dell'energia, Doctoral thesis - University Sapienza, 2007.

[3] Marco Sala, Nelli Ceccherini, Schermature solari, Alinea, 2000.

[4] AA. VV., TRNSYS: A Transient System Simulation Program. Solar Energy Madison, 1997.

[5] M. Santamouris, D. Asimakopolous, Passive cooling of building, 1996. 